\title{
Mitochondrial Cumulative Damage Induced by Mitoxantrone: Late Onset Cardiac Energetic Impairment
}

\author{
Luciana Grazziotin Rossato - Vera Marisa Costa $\cdot$ Eliane Dallegrave • \\ Marcelo Arbo $\cdot$ Renata Silva $\cdot$ Rita Ferreira $\cdot$ Francisco Amado • \\ Ricardo Jorge Dinis-Oliveira $\cdot$ José Alberto Duarte $\cdot$ Maria de Lourdes Bastos \\ Carlos Palmeira $\cdot$ Fernando Remião
}

Published online: 5 October 2013

(c) Springer Science+Business Media New York 2013

\begin{abstract}
Mitoxantrone (MTX) is a chemotherapeutic agent, which presents late irreversible cardiotoxicity. This work aims to highlight the mechanisms involved in the MTX-induced cardiotoxicity, namely the effects toward mitochondria using in vivo and in vitro studies. Male Wistar rats were treated with 3 cycles of $2.5 \mathrm{mg} / \mathrm{kg}$ MTX at day 0,10 , and 20 . One treated group was euthanized on day 22 (MTX22) to evaluate the early MTX cardiac toxic effects, while the other was euthanized on day 48 (MTX48), to allow the evaluation of MTX late cardiac effects. Cardiac mitochondria isolated from 4 adult untreated rats were also used to evaluate in vitro the MTX $(10 \mathrm{nM}, 100 \mathrm{nM}$, and $1 \mu \mathrm{M})$ direct effects upon mitochondria functionality. Two rats of MTX48 died on day 35, and MTX treatment caused a reduction in relative body weight gain in both treated groups with no significant
\end{abstract}

L. G. Rossato $(\bowtie) \cdot$ V. M. Costa · E. Dallegrave · M. Arbo ·

R. Silva · R. J. Dinis-Oliveira - M. de Lourdes Bastos ·

F. Remião $(\bowtie)$

REQUIMTE, Laboratório de Toxicologia, Departamento de Ciências Biológicas, Faculdade de Farmácia, Universidade do Porto, Porto, Portugal

e-mail: luciana.g.rossato@gmail.com

F. Remião

e-mail: remiao@ff.up.pt

E. Dallegrave

Universidade Federal de Ciências da Saúde de Porto Alegre,

Porto Alegre, Brazil

R. Ferreira

QOPNA, Departamento de Química, Universidade de Aveiro, Aveiro, Portugal

F. Amado

Escola Superior de Saúde, Universidade de Aveiro (ESSUA),

Aveiro, Portugal changes in water and food intake. Decreased levels of plasma total creatine kinase and CK-MB were detected in the MTX22 group, and increased plasma levels of lactate were seen in MTX48. Increased cardiac relative mass and microscopic changes were evident in both treated groups. Considering mitochondrial effects, for the first time, it was evidenced that MTX induced an increase in the complex IV and complex $\mathrm{V}$ activities in MTX22 group, while a decrease in the complex $\mathrm{V}$ activity was accompanied by the reduction in ATP content in the MTX48 rats. No alterations in mitochondria transmembrane potential were found in isolated mitochondria from MTX48 rats or in isolated mitochondria directly incubated with MTX. This study highlights the relevance of the cumulative MTX-induced in vivo mitochondriopathy to the MTX cardiotoxicity.

\section{R. J. Dinis-Oliveira}

Departamento de Medicina Legal e Ciências Forenses,

Faculdade de Medicina, Universidade do Porto, Porto, Portugal

\section{R. J. Dinis-Oliveira}

Department of Sciences, Advanced Institute of Health Sciences North, CESPU, CRL, Gandra, Portugal

J. A. Duarte

CIAFEL, Faculdade de Desporto, Universidade do Porto, Porto, Portugal

C. Palmeira

Centro de Neurociências e Biologia Celular de Coimbra, Departamento de Ciências da Vida, Universidade de Coimbra, Coimbra, Portugal 
Keywords Mitoxantrone - Cardiotoxicity ·

Mitochondria

\begin{tabular}{|c|c|}
\hline \multicolumn{2}{|c|}{ Abbreviations } \\
\hline ATP & Adenosine $5^{\prime}$-triphosphate \\
\hline AST & Aspartate aminotransferase \\
\hline BN-PAGE & $\begin{array}{l}\text { Blue native polyacrylamide gel } \\
\text { electrophoresis }\end{array}$ \\
\hline CK & Creatine kinase \\
\hline DAB & Diaminobenzidine \\
\hline DTNB & 5,5-Dithio-bis(2-nitrobenzoic acid) \\
\hline GSH & Reduced glutathione \\
\hline GSHt & Total glutathione \\
\hline GSSG & Oxidized glutathione \\
\hline $\mathrm{HClO}_{4}$ & Perchloric acid \\
\hline LDH & Lactate dehydrogenase \\
\hline MTX & Mitoxantrone \\
\hline$\beta$-NADPH & $\begin{array}{l}\text { Reduced } \beta \text {-nicotinamidephosphate adenine } \\
\text { dinucleotide }\end{array}$ \\
\hline $\mathrm{TPP}^{+}$ & Tetraphenylphosphonium \\
\hline
\end{tabular}

\section{Introduction}

Mitoxantrone (MTX) is an antitumor agent used in the treatment of breast and prostate cancer, acute leukemia, lymphoma, and in the treatment of multiple sclerosis due to its immunosuppressive properties [1, 2]. However, the cumulative cardiotoxic profile of MTX is well known, and it can affect up to $18 \%$ of treated patients [1]. The MTXinduced cardiotoxicity is irreversible, dose-dependent, and it may occur years after treatment [3]. Clinically, MTX cardiotoxicity is mainly characterized by a reduction in left ventricular stroke volume and congestive heart failure [1,3].

The risk of MTX-induced cardiac side effects increases greatly with cumulative dose up to $140 \mathrm{mg} / \mathrm{m}^{2}$ [2, 3] Human mean maximum MTX plasma concentrations are about 1.5 and $12 \mu \mathrm{M} 30 \mathrm{~min}$ after 15 or $90 \mathrm{mg} / \mathrm{m}^{2}$ intravenous infusion, respectively [4]. The accumulation of MTX in the heart tissue is well documented [5, 6], with described concentrations around $716 \mathrm{ng} / \mathrm{g}$ wet tissue 35 days after MTX single dose of $12 \mathrm{mg} / \mathrm{m}^{2}$ [6]. Furthermore, we highlighted that at least one biotransformation product of MTX, the naphthoquinoxaline metabolite (described as toxic), also accumulates in the rat heart, evidencing a prolonged exposure of the cardiac cells to MTX and this bioactive product [7].

Recently, we showed that therapeutic concentrations of MTX $(100 \mathrm{nM}$ and $1 \mu \mathrm{M})$ caused an important energetic imbalance evidenced by decreased ATP levels, hyperpolarization of the mitochondrial membrane potential, and a rise in the intracellular calcium levels after 24, 48, and $96 \mathrm{~h}$ of MTX incubation in a cardiomyoblast cell model (H9c2 cells) [8]. Additionally, MTX caused late inhibition of ATP-synthase expression and activity with concomitant increase in the reactive species generation after $96 \mathrm{~h}$ incubation with both previously referred concentrations [8]. In other studies, energetic imbalance was also observed in cultured neonatal rat heart cells after MTX incubation [9], and mitochondrial swelling was described in female BALB/c mice heart after MTX treatment [10]. Furthermore, uncoupling of oxidative phosphorylation was described in cardiac mitochondria isolated from rats treated with MTX [11]. Even so, studies to elucidate the mechanisms involved in the cardiotoxicity of MTX in vivo are scarce, especially with focus on mitochondrial functionality. Thus, the present work aims to contribute to the better understanding the in vivo MTX-induced cumulative cardiotoxicity using male adult Wistar rats subjected to 3 cycles of MTX treatment. MTX total cumulative dose was $7.5 \mathrm{mg} / \mathrm{kg}$, which corresponds to $48 \mathrm{mg} / \mathrm{m}^{2}$ by the allometric relationship, and therefore a clinical therapeutic dose. Early and late toxic effects ( 2 vs. 28 days after reaching the MTX total cumulative dose) were evaluated in order to assess the maladaptive responses involved in the MTX-induced cardiotoxicity. Furthermore, a complementary in vitro study, using rat-isolated cardiac mitochondria, was performed to understand the direct effects of MTX in the cardiac mitochondrial function.

\section{Materials and Methods}

\section{Chemicals}

All chemicals were of analytical grade. MTX hydrochloride, reduced glutathione (GSH), oxidized glutathione (GSSG), glutathione reductase (GR, EC 1.6.4.2), 2-vinylpyridine, reduced $\beta$-nicotinamide phosphate adenine dinucleotide ( $\beta$-NADPH), adenosine $5^{\prime}$-triphosphate (ATP), cytochrome $\mathrm{c}$, defatted bovine serum albumin, subtilopeptidase A type VIII, 5,5-dithio-bis(2-nitrobenzoic acid) (DTNB), luciferin, luciferase, subtilisin A protease, diaminobenzidine (DAB), glutaraldehyde, formaldehyde, and osmium tetroxide were purchased from Sigma-Aldrich (St. Louis, MO). HMWnative marker was purchased from GE Healthcare, Buckinghamshire, UK. Perchloric acid $\left(\mathrm{HClO}_{4}\right)$ and propylene oxide were obtained from Merck (Darmstadt, Germany). The reagents for the creatine kinase (CK), CK-MB, and lactate analysis were purchased from PZ Cormay S.A.

\section{Animals}

For the in vivo study, 15 adult male Wistar rats (Charles River Laboratories, Barcelona, Spain) weighing 240-300 g were used. Four additional male Wistar rats, weighting 
200-250 g, were utilized for the in vitro study. The animals were housed in cages, with a temperature and humidity controlled environment. Food and water were provided ad libitum, and animals were subjected to a $12 \mathrm{~h}$ light-dark cycle. Animal experiments were approved by the Ethical Committee of Faculty of Pharmacy of the University of Porto (protocol number 09/04/2013). Housing and experimental treatment of the animals were in accordance with the Guide for the Care and Use of Laboratory Animals from the Institute for Laboratory Research. The experiments complied with current Portuguese laws.

\section{Dose Regimen}

For 1 week prior to the in vivo experiment, animals were acclimatized in cages. Animals were distributed into 3 groups (5 animals per group): control, MTX22, and MTX48; however, in order to allow individual measurements of food/ water consumption, rats were distributed as 1 animal per cage. Animals were treated with 3 cycles by intraperitoneal route $(5 \mathrm{ml} / \mathrm{kg}$ ), of saline solution $(0.9 \% \mathrm{NaCl})$ (control) or MTX $2.5 \mathrm{mg} / \mathrm{kg}$ (MTX22 and MTX48) on day 0,10 , and 20, the MTX-treated groups reaching a total cumulative dose of $7.5 \mathrm{mg} / \mathrm{kg}$ on day 20 . The dose was calculated taking into account previously pilot studies and the body surface area of the rats in order to correlate the dose in this species considering human top limit of the commonly used doses [5]. Thus, $2.5 \mathrm{mg} / \mathrm{kg}$ corresponds to $16 \mathrm{mg} / \mathrm{m}^{2}$ of a rat weighing $240 \mathrm{~g}$, being similar to the dose administered in humans and about one-tenth of the maximum dose allowed in humans $(140 \mathrm{mg} /$ $\mathrm{m}^{2}$ ) [1]. The regimen of administration (1 cycle every 10 days) was performed aiming to simulate the human chemotherapeutic cycles. The interval of 10 days was defined taking into account the life cycle of the rat and the clinical conditions observed in pilot studies.

During the experiment, daily clinical evaluations of all animals were performed by the veterinary doctor of the team. The parameters evaluated were as follows: piloerection, dehydration, hemorrhage and diarrhea, motor function (tone and movement coordination), breathing (rate and depth, gasping), mucosal color (pale, cyanotic), and clinical signals of abdominal pain. The individual weight and consumption of food and water were also evaluated daily until day 30 .

The MTX22 group was euthanized on day 22, in order to assess the MTX-induced cumulative damage 2 days after the last cycle of treatment. MTX48 group was euthanized on day 48 , i.e., 28 days after the last cycle of treatment, in order to evaluate the late cumulative responses. Animals were anesthetized with xylazine/ketamine $(10-100 \mathrm{mg} / \mathrm{kg}$ ), and blood was collected through cardiac puncture. All the procedures were equally performed for control and treated rats.
Plasma Biochemical Analysis

Blood was collected into heparinized tubes. Plasma levels of aspartate aminotransferase (AST), CK, CK-MB, and lactate were evaluated in duplicate on an AutoAnalyzer (PRESTIGE ${ }^{\circledR} 24 \mathrm{i}$, PZ Cormay S.A.) using the respective kits and following the manufacturer instructions.

Tissue Preparation for Light and Transmission Electron Microscopy

For light microscopy, heart (right and left ventricle) was sliced in $2-4 \mathrm{~mm}^{3}$ pieces, approximately, and fixed in $4 \%$ formaldehyde $\left(10-20 \mathrm{~h}, 4{ }^{\circ} \mathrm{C}\right)$, which were further dehydrated with graded ethanol (Panreac, Barcelona, Spain) concentrations and mounted in paraffin (MERK, Darmstadt, Germany) following routine standard procedures. Semi-thin sections $(5 \mu \mathrm{m})$ were cut in a microtome (Leica Microsystems, Model RM2125) and mounted on silanecoated slides (Sigma, S4651-72EA). After dewaxed in xylene and hydrated through graded alcohol concentrations, tissue sections were stained with haematoxylin-eosin (Atom Scientific Ltd, England) and analyzed under a light microscope coupled to a digital camera (Axio Imager A1, Carl Zeiss, Oberkochen, Germany).

For transmission electron microscopy, $1 \mathrm{~mm}^{3}$ right and left ventricle pieces were fixed in $2 \%$ glutaraldehyde $(2 \mathrm{~h}$, $4{ }^{\circ} \mathrm{C}$ ), post-fixed with $2 \%$ osmium tetroxide, dehydrated in graded ethanol and later embedded in Epon (TAAB 812 Resin, Kit Cat. No. T024), using routine standard procedures. Ultrathin $(100 \mathrm{~nm})$ sections obtained in an ultramicrotome (Reichert Ultracut) were mounted in copper grids (300 Mesh, from TAAB Laboratories Equipment Ltd, England) and further contrasted with uranyl acetate and lead citrate for transmission electron microscopy analysis (Zeiss EM 10A) at an accelerating voltage of $60 \mathrm{kV}$.

Isolation of Cardiac Mitochondria for the Evaluation of Complexes IV and V Activities and Cardiac Mitochondrial Content

Cardiac mitochondria from control and MTX-treated rats were isolated for the evaluation of in-gel activity of complexes IV and V and cardiac mitochondrial content. Hearts were minced with scissors in homogenization buffer $[250 \mathrm{mM}$ sucrose, $10 \mathrm{mM}$ HEPES, $0.5 \mathrm{mM}$ EGTA (pH 7.4)], supplemented with subtilpeptidase A type VIII (1 mg/g tissue). Following $10 \mathrm{~min}$ of incubation on ice, albumin fat-free was added to a final concentration of $10 \mathrm{mg} / \mathrm{mL}$. The tissue was subsequently rinsed three times with buffer, homogenized with a tightly fitted Potter-Elvehjen homogenizer and Teflon pestle, and centrifuged $\left(14,500 \mathrm{~g}, 10 \mathrm{~min}, 4^{\circ} \mathrm{C}\right)$. The resulting pellet was resuspended in homogenization buffer free of 
enzyme, homogenized, and centrifuged $\left(750 \mathrm{~g}, 10 \mathrm{~min}, 4^{\circ} \mathrm{C}\right)$. Mitochondria-enriched pellet was obtained by centrifuging the resulting supernatant $\left(12,500 \mathrm{~g}, 20 \mathrm{~min}, 4^{\circ} \mathrm{C}\right)$, washed twice, and then aliquoted for subsequent analysis [12]. Protein content was determined with RC DC Protein Assay kit (BioRad, Hercules, CA, USA) using bovine serum albumin as standard.

\section{Blue Native (BN)-PAGE Separation of Mitochondria Membrane Complexes}

The BN-PAGE separation of mitochondrial membrane complexes was performed as already described [12] using 4 animals per group. Mitochondria (200 $\mu \mathrm{g}$ of protein) were pelleted by centrifugation $\left(20,000 \mathrm{~g}, 10 \mathrm{~min}, 4^{\circ} \mathrm{C}\right)$ and then resuspended in solubilization buffer $(50 \mathrm{mM} \mathrm{NaCl}, 50 \mathrm{mM}$ imidazole, $2 \mathrm{mM}$ 6-aminohexanoic acid, $1 \mathrm{mM}$ EDTA pH 7.0) with $1 \%(\mathrm{w} / \mathrm{v})$ digitonin. After $10 \mathrm{~min}$ on ice, insoluble material was removed by centrifugation $(20,000 \mathrm{~g}$, $20 \mathrm{~min}, 4^{\circ} \mathrm{C}$ ). Soluble components were combined with $0.5 \%(\mathrm{w} / \mathrm{v})$ Coomassie Blue G250, $50 \mathrm{mM} \varepsilon$-amino $n$ caproic acid, and $4 \%(\mathrm{w} / \mathrm{v})$ glycerol and separated on a 4-13\% gradient acrylamide gradient gel with $3.5 \%$ sample gel on top. Anode buffer contained $25 \mathrm{mM}$ imidazole $\mathrm{pH}$ 7.0. Cathode buffer $(50 \mathrm{mM}$ tricine and $7.5 \mathrm{mM}$ imidazole $\mathrm{pH} 7.0)$ containing $0.02 \%(\mathrm{w} / \mathrm{v})$ Coomassie Blue G250 was used during $1 \mathrm{~h}$ at $70 \mathrm{~V}$, the time needed for the dye front to reach approximately one-third of the gel. Cathode buffer was then replaced for one containing only $0.002 \%(w / v)$ Coomassie Blue G250, and the native complexes were separated at $200 \mathrm{~V}$ for $4 \mathrm{~h}$ at $4{ }^{\circ} \mathrm{C}$. A native protein standard HMW-native marker (GE Healthcare) was used. The gels were stained with Coomassie Colloidal for protein visualization and scanned with Gel Doc XR System (Bio-Rad, Hercules, CA, USA). Band detection, quantification, and matching were performed using QuantityOne Imaging software (v4.6.3, Bio-Rad). Mitochondrial DNA was quantified using the Qubit $^{\circledR}$ dsDNA BR assay kit (Invitrogen, Carlsbad, CA, USA). Samples were read in a Qubit ${ }^{\circledR}$ 2.0 Fluorometer, and results were expressed as the ratio between the mitochondrial DNA concentration and the heart mass.

\section{In-Gel Activity of Mitochondrial Complexes IV and V}

The in-gel activity of complexes IV and V was determined using the methods described elsewhere [12]. Briefly, complex IV-specific heme stain in BN-PAGE gels was determined using $10-\mu \mathrm{L}$ horse-heart cytochrome c $(5 \mathrm{mM})$ and $0.5 \mathrm{mg}$ DAB dissolved in $1 \mathrm{ml}$ of $50 \mathrm{mM}$ sodiumphosphate, $\mathrm{pH} 7.2$. The reaction was stopped by $50 \%(\mathrm{v} / \mathrm{v})$ methanol and $10 \%(\mathrm{v} / \mathrm{v})$ acetic acid, and the gels were then transferred to water.
ATP hydrolysis activity of complex $\mathrm{V}$ was analyzed by incubating the native gels with $35 \mathrm{mM}$ Tris, $270 \mathrm{mM}$ glycine buffer, $\mathrm{pH} 8.3$ at $37{ }^{\circ} \mathrm{C}$, supplemented with $14 \mathrm{mM}$ $\mathrm{MgSO}_{4}, 0.2 \%(\mathrm{w} / \mathrm{v}), \mathrm{Pb}\left(\mathrm{NO}_{3}\right)_{2}$, and $8 \mathrm{mM}$ ATP. Lead phosphate precipitation is proportional to the enzymatic ATP hydrolysis activity. The reaction was stopped by incubation with $50 \%(\mathrm{v} / \mathrm{v})$ methanol for $30 \mathrm{~min}$, and the gels were then transferred to water.

Gels were scanned with Gel Doc XR System (Bio-Rad, Hercules, CA, USA). Band quantification was performed using QuantityOne Imaging software (v4.6.3, Bio-Rad).

Determinations in the Cardiac Tissue

After the excision of hearts, they were washed in a phosphate-saline buffer solution ( $\mathrm{pH}$ 7.4), dried, and weighted to assess the relative mass of the heart (calculated as a percentage of the total body weight on the day of euthanasia). Afterward, heart tissue samples were homogenized $[1: 4(\mathrm{~m} / \mathrm{v})]$ in ice-cold phosphate buffer solution (pH 7.4), with an Ultra-Turrax ${ }^{\circledR}$ homogenizer and centrifuged $\left(3,000 \mathrm{~g}, 10 \mathrm{~min}, 4^{\circ} \mathrm{C}\right)$. Aliquots were taken to determine the glutathione status, ATP levels, and total protein levels.

\section{Total Glutathione (GSHt), GSH, and GSSG Levels in the Cardiac Tissue}

An aliquot of the supernatant referred previously was added to an equal volume of $\mathrm{HClO}_{4} 10 \%(5 \%$ as final concentration). Samples were again homogenized and centrifuged $\left(16,000 \mathrm{~g}, 10 \mathrm{~min}, 4^{\circ} \mathrm{C}\right)$, and the supernatant was used to determinate the glutathione status [13]. The GSHt and GSSG levels were evaluated by the DTNBGSSG reductase recycling assay, as previously described $[14,15]$. Briefly, for GSHt quantification, $200 \mu \mathrm{L}$ of supernatant was neutralized with $200 \mu \mathrm{L}$ of $0.76 \mathrm{M}$ $\mathrm{KHCO}_{3}$ and centrifuged $\left(16,000 \mathrm{~g}, 5 \mathrm{~min}, 4{ }^{\circ} \mathrm{C}\right)$. For GSSG quantification, $10 \mu \mathrm{L}$ of 2-vinylpyridine was added to $200 \mu \mathrm{L}$ of acid supernatant and shaken during $1 \mathrm{~h}$ on ice prior to the neutralization step. In 96-well plates, $100 \mu \mathrm{L}$ of sample, standard, or blank were added in triplicate and mixed with $65 \mu \mathrm{L}$ of fresh reagent solution containing DTNB and $\beta$-NADPH. Plates were incubated at $30{ }^{\circ} \mathrm{C}$ for $15 \mathrm{~min}$ in a plate reader (PowerWaveX, BioTek Instruments) prior to the addition of $40-\mu \mathrm{L}$ glutathione reductase solution $(10 \mathrm{U} / \mathrm{mL})$. The final product of this reaction is a colored substance, and its formation was monitored for $3 \mathrm{~min}$, at $415 \mathrm{~nm}$, and compared with a standard curve. GSH and GSSG standard solutions were prepared in $\mathrm{HClO}_{4} 5 \%$ [15]. Results were expressed as $\mathrm{nmol} / \mathrm{mg}$ protein. 


\section{Cardiac ATP Levels}

An aliquot of supernatant obtained in "Determinations in the Cardiac Tissue" section was added to an equal volume of $\mathrm{HClO}_{4} 10 \%$ (5\% as final concentration). Samples were again homogenized and centrifuged $(16,000 \mathrm{~g}, 10 \mathrm{~min}$, $4{ }^{\circ} \mathrm{C}$ ), and the supernatant was used to determinate the ATP levels as described before [8]. One hundred $\mu \mathrm{L}$ of tissue homogenate was neutralized with $100 \mu \mathrm{L}$ of $0.76 \mathrm{M}$ $\mathrm{KHCO}_{3}$ and centrifuged $\left(16,000 \mathrm{~g}, 10 \mathrm{~s}, 4{ }^{\circ} \mathrm{C}\right)$. The ATP levels were quantified by the bioluminescence test after the reaction of $100-\mu \mathrm{L}$ neutralized supernatant with luciferin/ luciferase solution. ATP standards were prepared in $5 \%$ $\mathrm{HClO}_{4}$. ATP intracellular contents were normalized to the total protein content (nmol/mg of protein).

\section{Cardiac Total Protein Quantification}

An aliquot of the supernatant obtained in the "Determinations in the Cardiac Tissue" section was diluted in $0.3 \mathrm{M}$ $\mathrm{NaOH}$ and was used to assess the total protein levels in the cardiac tissue. The protein levels were determined, by a procedure based in the Lowry method, spectrophotometrically $(750 \mathrm{~nm})$, and using a microplate reader as previously described $[14,16]$.

Evaluation of the Mitochondrial Membrane Potential After In Vivo and In Vitro Treatment with MTX

Cardiac mitochondria from control and MTX48 rats were isolated for the assessment of the late cumulative effects induced by MTX toward the mitochondrial membrane potential. Moreover, an in vitro study with mitochondria isolated from 4 untreated rat hearts (not treated with MTX), which were euthanized through cervical dislocation, was also performed in order to assess the direct MTX effects in the mitochondrial function after incubation with MTX $(10 \mathrm{nM}, 100 \mathrm{nM}$, and $1 \mu \mathrm{M})$.

\section{Isolation of Cardiac Mitochondria}

Hearts were minced with scissors in homogenization buffer (250 mM sucrose, $1 \mathrm{mM}$ EGTA, HEPES-KOH $5 \mathrm{mM}$, pH 7.4 , and $0.1 \%$ fat-free bovine serum albumin) supplemented with $0.5 \mathrm{mg} / \mathrm{g}$ tissue of protease. Samples were homogenized using a Potter-Elvehjen homogenizer for $30 \mathrm{~s}$. The suspension was then incubated on ice for $1 \mathrm{~min}$, and the homogenization step was repeated for more $30 \mathrm{~s}$. After centrifugation $\left(11,000 \mathrm{~g}, 10 \mathrm{~min}, 4^{\circ} \mathrm{C}\right)$, the pellet was gently homogenized in the homogenization buffer and centrifuged $\left(800 \mathrm{~g}, 10 \mathrm{~min}, 4{ }^{\circ} \mathrm{C}\right)$. The obtained supernatant was centrifuged $\left(12,000 \mathrm{~g}, 10 \mathrm{~min}, 4{ }^{\circ} \mathrm{C}\right)$, and the mitochondria pellet was washed twice with homogenization buffer without EGTA and bovine serum albumin. Protein content was determined through the Biuret method.

\section{Mitochondrial Transmembrane Potential Measurement}

The mitochondrial membrane potential was measured using an ion-selective electrode to measure the distribution of the tetraphenylphosphonium $\left(\mathrm{TPP}^{+}\right)$, as described before [17]. The reference electrode used was a $\mathrm{Ag} / \mathrm{AgCl}_{2}$, and mitochondria $(1 \mathrm{mg})$ were incubated with $1 \mathrm{ml}$ respiratory medium $(130 \mathrm{mM}$ sucrose, $50 \mathrm{mM} \mathrm{KCl}, 2.5 \mathrm{mM}$ $\mathrm{KH}_{2} \mathrm{PO}_{4}, 5$ mM HEPES, $\mathrm{pH}$ 7.4), supplemented with $2 \mu \mathrm{M}$ $\mathrm{TPP}^{+}$and $1.5 \mathrm{mM}$ rotenone, with constant stirring, at $25{ }^{\circ} \mathrm{C}$. MTX $(10 \mathrm{nM}, 100 \mathrm{nM}$, or $1 \mu \mathrm{M})$ was added to the incubation chamber. Mitochondria were energized by adding $5 \mathrm{mM}$ succinate [18]. The electrode was calibrated with $\mathrm{TPP}^{+}$assuming Nerstian distribution of the ion across the synthetic membrane. The mitochondrial membrane potential was expressed in $\mathrm{mV}$.

\section{Statistical Analysis}

Results are presented as mean \pm standard deviation. The evaluations of the rat relative body weight gain and the consumptions of food and water were followed daily; thus, the statistical analysis was performed using repeated measures ANOVA followed by the Student-NewmanKeuls post hoc test. In the other experiments, statistical comparisons between groups were performed with oneway ANOVA (in case of normal distribution) or KruskalWallis test (one-way ANOVA on ranks-in case of not normal distribution). Significance was accepted at $p$ values $<0.05$.

\section{Results}

Cumulative signs of Toxicity After MTX Treatment

On the day 22 ( 2 days after the last MTX cycle), 2 rats from the MTX22 group presented moderate piloerection. On the day 25 (5 days after the last MTX cycle), 3 animals from the group MTX48 showed moderate signals suggestive of toxicity, namely slight dehydration, cyanosis, and slight bleeding. In the following days, 1 of these animals fully recovered, but the other 2 died on the day 35 . The 2 remaining rats from MTX48 group did not show any significant clinical alteration during all time course of the study. As it can be seen in the Fig. 1, animals treated with MTX started to decrease the relative body weight gain compared to control group since the second cycle of drug administration. These decreases were statistically significant after the third cycle, when the animals treated with 


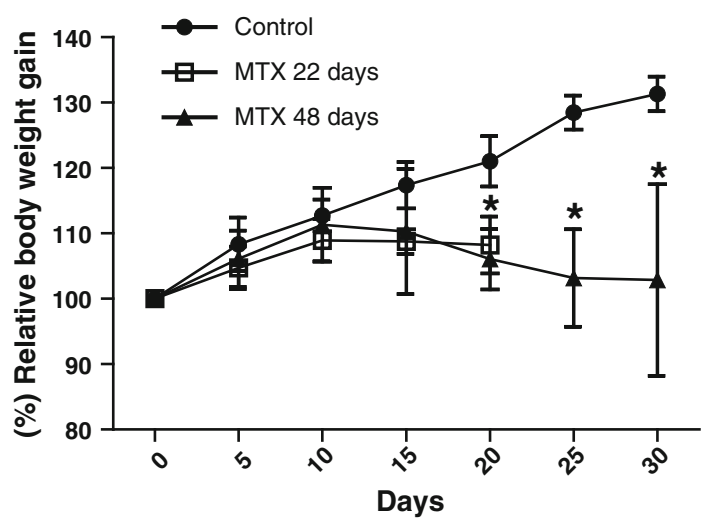

Fig. 1 Relative body weight $(\%)$ gain of the animals treated with MTX ( 3 cycles of $2.5 \mathrm{mg} / \mathrm{kg}$ ) and control group. Results are presented as mean \pm standard deviation. Statistical analysis was performed using repeated measures ANOVA followed by the Student-NewmanKeuls post hoc test $(* p<0.05$ vs. control)

MTX reached a cumulative dose of $7.5 \mathrm{mg} / \mathrm{kg}$, until the day 30 . This change is indicative of toxicity, and it was not accompanied by significant alterations in food or water consumptions (data not shown).

\section{Plasma Changes Induced by MTX Treatment}

As demonstrated in Table 1, plasma biochemical analysis revealed a significant transient decrease in the CK levels in the MTX22 group when compared to MTX48 group and control group. Conversely, the CK-MB isoenzyme presented the same trend: a significant decrease in the MTX22 group versus control. Values similar to control levels were found in the MTX48 group. The plasma lactate levels significantly increased in the MTX48 group compared to MTX22 and control groups (Table 1). No significant changes were observed in the plasma AST levels in MTXtreated groups compared to control animals (data not shown)

Table 1 Plasma biochemical parameters of the animals treated with MTX (3 cycles of $2.5 \mathrm{mg} / \mathrm{kg}$ ) and control group

\begin{tabular}{llcl}
\hline $\begin{array}{l}\text { Plasma } \\
\text { biomarker }\end{array}$ & \multicolumn{1}{l}{ Group } & \multicolumn{1}{l}{ MTX42 } & \multicolumn{1}{l}{ MTX48 } \\
\cline { 2 - 4 } & Control & \multicolumn{1}{l}{ MTX22 } & \\
\hline CK (U/L) & $537.8 \pm 195.6$ & $326.5 \pm 71.0^{*}$ & $441.3 \pm 26.9 \mathrm{~ns}$ \\
CK/MB (U/L) & $572.8 \pm 125.9$ & $390.3 \pm 58.7^{*}$ & $595.6 \pm 151.2 \mathrm{~ns}$ \\
$\begin{array}{l}\text { Lactate } \\
(\mathrm{mmol} / \mathrm{L})\end{array}$ & $1.3 \pm 0.4$ & $1.6 \pm 0.3 \mathrm{~ns}$ & $2.6 \pm 1.7^{*}$ \\
\hline
\end{tabular}

Results are presented as mean \pm standard deviation. Statistical analysis was performed using one-way ANOVA followed by the Student-Newman-Keuls post hoc test $(* p<0.05$ vs. control)

ns not significant

$* p<0.05$ vs. control
Microscopic Changes in the Cardiac Structure After MTX Treatment

Structural and ultrastructural analysis from control group revealed a preserved cellular and tissue morphology (data not shown). However, as shown in the Fig. 2, in both MTX-treated hearts, light microscopy revealed dispersed cellular and interstitial edema, diffusion of inflammatory cells, and proliferation of connective tissue (A and B). In the MTX48 group, dispersed areas of a more intense proliferation of the connective tissue with abundant cellular infiltration, probably by fibroblasts, and signs of obstruction in some small caliber arteries (A and B) were seen. The transmission electron microscopy revealed edema of cardiomyocytes (C, D, and E), lysosome activation in the cardiomyocytes, proliferation of connective tissue (F and E) and fibroblasts, and occupation of the endothelial space by digiforme endothelial projections ( $\mathrm{C}$ and $\mathrm{E})$. In general, mitochondria showed clearly defined cristae, however, with abnormal orientations, but no signs of mitochondrial swelling were observed. Giant mitochondria with aberrant morphology $(\mathrm{C}, \mathrm{D}$, and $\mathrm{E})$ were frequently interspaced with normal morphology and dimensions ones. Abundant collagen deposition in the interstitial space was frequently observed in MTX48 (D and E). It is important to refer that the cardiac damage shown in the histology was observed in both right and left ventricles, suggesting that both sides were equally affected by MTX. The sub-endocardial region was more affected compared to the sub-pericardial since the cellular edema and fibrosis were more pronounced in this region.

\section{Cardiac Alterations Induced by MTX}

The cardiac relative mass (\%) was evaluated as an indicator of cardiotoxicity (Fig. 3). The animals, which were euthanized on the day 22 (MTX22), did not show any changes in the cardiac relative mass $(0.27 \pm 0.02 \%)$ compared to control group $(0.27 \pm 0.03 \%)$. However, the cardiac relative mass in the MTX48 group was significantly higher $(0.33 \pm 0.03 \%)$ when compared to control or MTX22 group, evidencing a late cardiac response (Fig. 3). These changes in the cardiac relative mass were not accompanied by alterations in the cardiac total protein levels (data not shown). No significant changes in the GSHt, GSH, or GSSG cardiac levels were observed in any of the end time points (data not shown).

Isolated cardiac mitochondria were used to evaluate the activity of complexes IV and V of the mitochondrial respiratory chain of the treated rats. As shown in the Fig. 4a), the organization pattern of the five respiratory chain complexes is in accordance with the described in the literature for heart tissue [12]. No significant qualitative or 

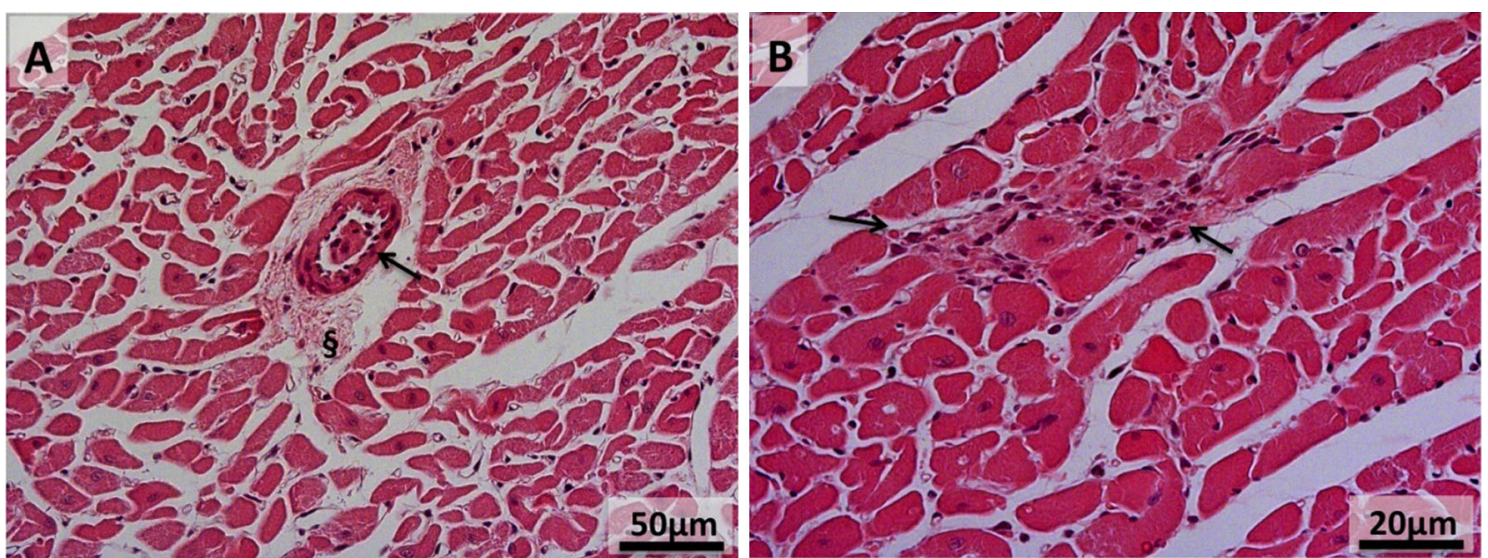

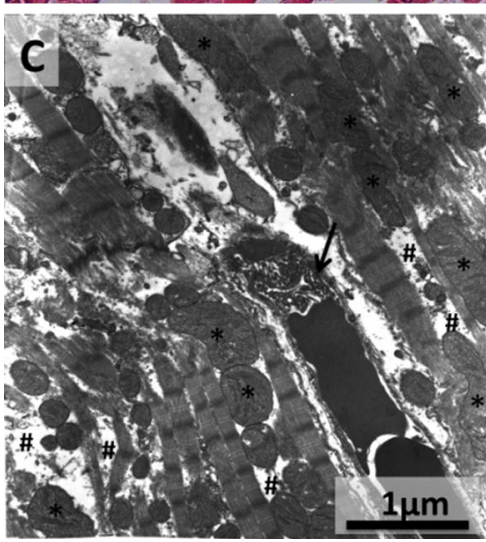

Fig. 2 Light $(\mathbf{a}, \mathbf{b})$ and electron micrographs $(\mathbf{c}, \mathbf{d}, \mathbf{e})$ of heart tissue sections representative of the common structural and ultrastructural modifications observed in MTX-treated animals. In a (MTX48), it can be observed an arteriole, with its lumen partially obstructed (arrow), surrounded by an area of connective tissue (§); a general enlargement of the interstitial space with dispersed swollen cardiomyocytes (with increased inter-myofibril space) is also depicted. A focal area of fibrosis (arrows) with abundant cellular infiltration is shown in

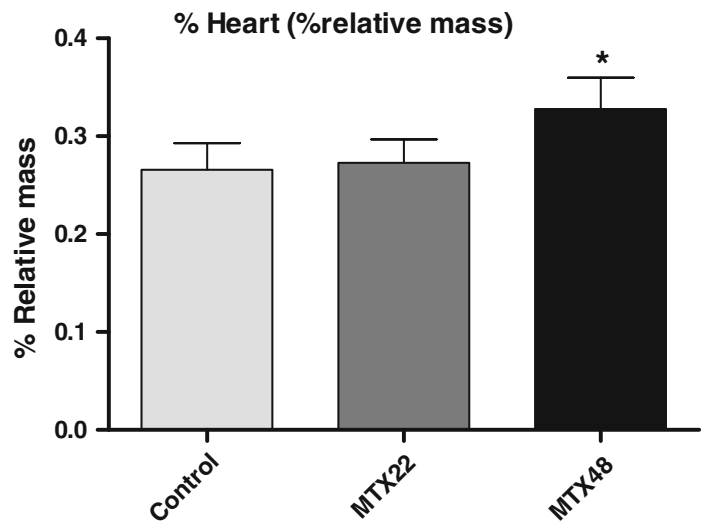

Fig. 3 Heart relative mass (\%) of the animals treated with MTX (3 cycles of $2.5 \mathrm{mg} / \mathrm{kg}$ ) and control group. Results are presented as mean \pm standard deviation. Statistical analysis was performed using one-way ANOVA followed by the Student-Newman-Keuls post hoc test $(* p<0.05$ vs. control) b (MTX48). At the ultrastructural level, an enlargement of intermyofibril space (\#) and several giant and/or aberrant mitochondria with abnormal cristae (asterisk) can be observed in c (MTX48), d (MTX48), and e (MTX48); capillaries with their lumen occupied by digiform structures are shown in $\mathbf{c}$ and $\mathbf{e}$ (arrow); interstitial collagen fibers are also illustrated in $\mathbf{d}$ and, with a special amount, in $\mathbf{e}(\S)$. The figures refer to the left ventricle; however, similar cardiac changes were found in the right ventricle

quantitative differences in the band pattern were observed between groups in the BN-PAGE. The in-gel activity of complex IV revealed significantly higher activity level in the cardiac mitochondria from rats treated with MTX $(155.8 \pm 33.6 \%$ for the MTX22 group, $148.3 \pm 14.4 \%$ for the MTX48 group vs. $100.0 \pm 2.8 \%$ for the control group) (Fig. 4b). Considering the complex V activity, a significant increase in MTX22 $(172.6 \pm 20.0 \%)$ and a significant decrease in MTX48 $(58.1 \pm 21.0 \%)$ were observed in comparison with the control $(100.0 \pm 8.3 \%$; Fig. 4c). Regarding cardiac ATP levels, a significant decrease in the MTX48 group $(7.8 \pm 1.5 \mathrm{nmol} / \mathrm{mg})$ compared to control $(16.0 \pm 2.8 \mathrm{nmol} / \mathrm{mg})$ or $\mathrm{MTX} 22$ $(17.6 \pm 6.7 \mathrm{nmol} / \mathrm{mg})$ groups was observed, as demonstrated in the Fig. 5. However, no significant changes in the ratio between mitochondrial DNA concentration and heart mass were found (data not shown). 


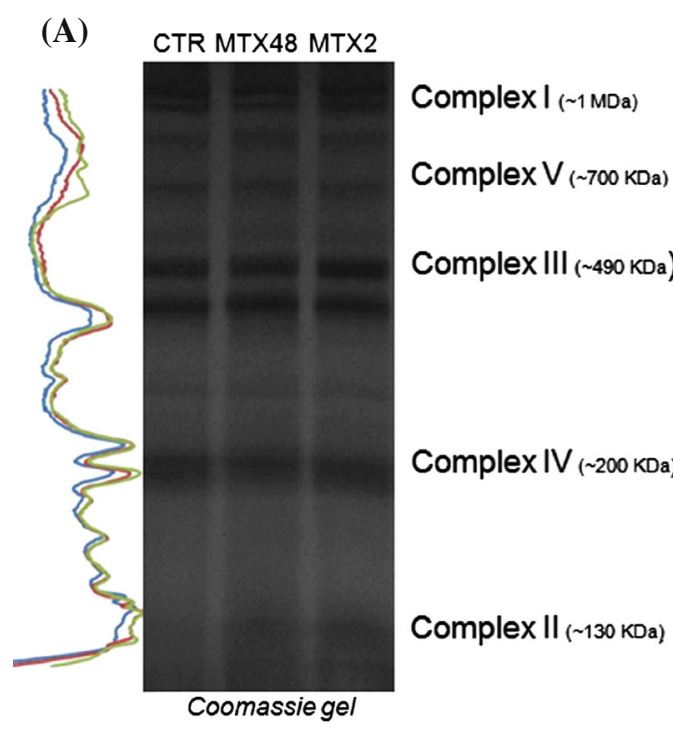

(B)
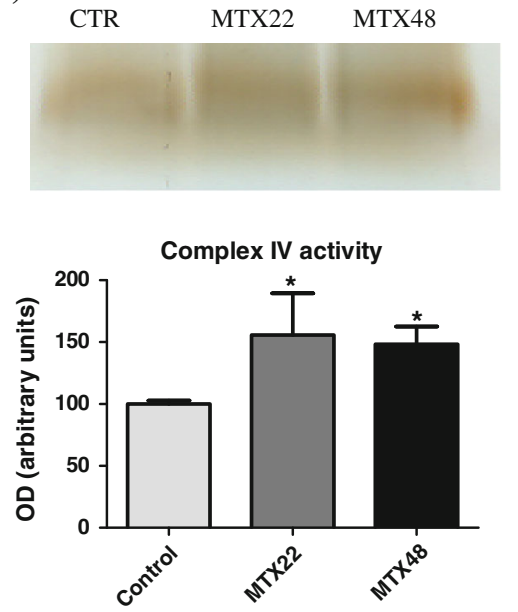

(C)
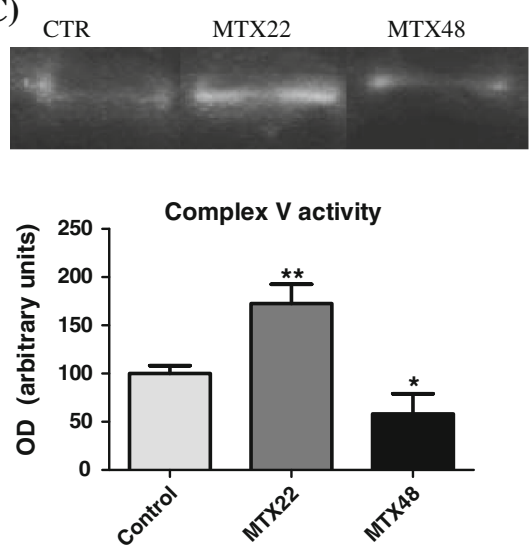

Fig. 4 a BN-PAGE profile of mitochondria isolated from control, MTX22, and MTX48 groups. An overlap of the density variation in the lanes of the 3 groups is presented on the left. b Representative image and graphic representation of histochemical staining of the ingel activity of complex IV. c Representative image and graphic

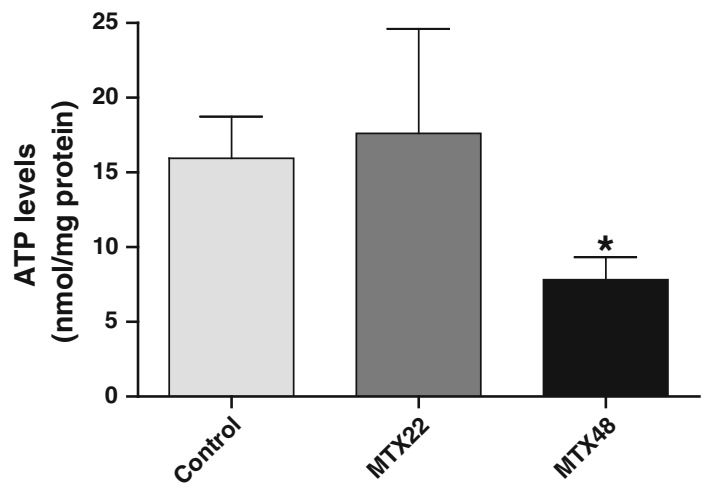

Fig. 5 Cardiac ATP levels of the animals treated with MTX (3 cycles of $2.5 \mathrm{mg} / \mathrm{kg}$ ) and control group. Results are presented as mean \pm standard deviation. Statistical analysis was performed using Kruskal-Wallis test (ANOVA on ranks) followed by the StudentNewman-Keuls post hoc test $\left({ }^{*} p<0.05\right.$ vs. control) representation of histochemical staining of the in-gel activity of complex V. Results are presented as mean \pm standard deviation. Statistical analysis was performed using Kruskal-Wallis test (ANOVA on ranks) followed by the Student-Newman-Keuls post hoc test $(* p<0.05$ vs. control)

Evaluation of the Transmembrane Potential in CardiacIsolated Mitochondria

Transmembrane potential sustained by mitochondria upon energization is essential for mitochondrial function. Given the previously presented results found in vivo, we investigated the possible mitochondrial deleterious effect of MTX using cardiac mitochondria isolated from MTX48 group. Altogether, no significant changes in the $\mathrm{TPP}^{+}$ assay were observed for the MTX48 mitochondria compared to control group. However, mitochondria from 2 animals presented an increased lag phase, demonstrating a difficulty to repolarize and sustain the mitochondrial potential (data not shown).

When mitochondria from untreated rats were incubated with 3 different MTX concentrations $(10 \mathrm{nM}, 100 \mathrm{nM}$, and $1 \mu \mathrm{M}$ ), energization phase (initial transmembrane 
potential, depolarization induced by the addition of ADP, repolarization, or lag phase) was not affected when compared to control (data not shown).

\section{Discussion}

In the present work, for the first time, the MTX-induced cumulative cardiotoxicity was ensued after the administration of reliable doses of MTX to rats evaluated at 2 different endpoints: 2 days after the last cycle of treatment (MTX22) and 28 days after the last MTX administration (MTX48). The cardiotoxicity of MTX was verified through the changes in the cardiac structure as showed by the transmission electron and optic microscopy (Fig. 2) and by the increases in the \% of relative heart mass per body weight observed in the MTX48 group (Fig. 3). The major contribution of this study was to show, for the first time, the different effects on mitochondrial respiratory chain with similar MTX dose and schedule regime of administration in vivo when animals were euthanized at two distinct endpoints. It became evident that mitochondrial function alterations were more pronounced in the later time point, as seen by the presence of aberrant mitochondria, changes of mitochondrial complexes IV and V activities, and depletion of cardiac ATP levels all in MTX48 animals. On the other hand, in MTX22 rats, increases in mitochondrial complexes IV and $\mathrm{V}$ was observed with no significant alteration in cardiac ATP levels when compared to control rat hearts.

The in vivo model employed in the present work seems to be appropriate to study cumulative toxicity induced by MTX treatment, since it reproduced human clinical observations, such as the late development of cardiotoxicity. This is an important point regarding the difficulties to pharmacologically induce a heart failure condition in laboratory animal models [19].

Although clinical aspects of doxorubicin and MTX are partially the same, the underlying mechanisms seem to differ. In fact, the MTX-induced cardiotoxicity may have its origin in its interference with cardiac energetic metabolism instead of oxidative stress [8, 10, 11, 20, 21]. In the present study, no observed significant alterations in the cardiac glutathione levels (GSHt, GSH, and GSSG) were found, which is in accordance with our previous in vitro results [8]. Even so, significant changes in ATP levels were found in MTX48 group. The sequence of events in the establishment of a failing and energy-starved heart usually follows a time course, first with decreases in the phosphocreatine levels, followed by the creatine loss, and, ultimately, by ATP depletion [22]. Decreases in the total CK activity and CK-MB, as observed in the MTX22 group
(Table 1), may be interpreted as a hallmark of the heart failure development [23]. However, all signaling pathways involved in CK alterations remain to be elucidated [23]. The development of late MTX-induced cardiotoxicity was characterized by the energetic depletion observed in the MTX48 group. It is well known that the loss of ATP in the failing myocardium is a slow and progressive phenomenon, only being detectable in the presence of a severe heart failure [22]. Plasma lactate levels increased in the MTX48 group in comparison with MTX22 and control levels (Table 1), and it was accompanied by depletion in the ATP cardiac levels and increase in the cardiac relative mass, suggesting a cardiac failure condition. Increased levels of plasma lactate, as observed in the MTX48 group (Table 1), are suggestive of lactic acidosis and occur in response to tissue hypoxia, uncoupling of oxidative phosphorylation, congestive heart failure, generalized cachexia (due to increased anaerobic glycolysis in the skeletal muscle), or in situations where the hepatic clearance is compromised [23, 24]. At MTX48, apparently, an energetic shift occurred, and the glycolytic pathway seems to be the major supplier of cardiac ATP, not being enough to warrant a healthy heart. In fact, when failing, the heart assumes the fetal pattern, switching the main energetic substrate source from fatty acids to glucose [23]. However, as efficiency of glucose oxidation is reduced, more lactate is generated [23], and the energetic impairment was evidenced by the depletion of the ATP cardiac levels in MTX48 rats (Fig. 5). In fact, it was described that failing human hearts have about 25-40 \% less ATP than healthy hearts, and hearts in energetic starvation fail to support an increase in the workload [22, 25]. Indeed, the length of time that the heart can survive with such ATP depletion still remains unclear [22]. The energetic imbalance caused by MTX was already demonstrated in vitro, after incubation of MTX with cultured neonatal cardiomyocytes isolated from rats [9] and with $\mathrm{H} 9 \mathrm{c} 2$ cells [8].

The late MTX-induced mitochondrial degeneration was also evidenced by aberrant mitochondrial morphology and subsequent functional studies. Microscopic changes in the cardiac mitochondrial architecture (namely cristolysis and swelling) were observed in vitro [26] and in vivo [10, 11] with MTX. In the present study, the mitochondrial cristae are well defined, but in many organelles, they are abnormally oriented, without signs of swelling. The mitochondrial morphologic changes are restricted to giant and aberrant aspect (Fig. 2). One hypothesis that could explain this finding is the MTX ability to intercalate into the DNA molecule causing double and single breaks and inhibiting DNA and RNA synthesis [27], which may compromise the mitochondrial turnover.

The activity of mitochondrial IV and V complexes was also evaluated in both time points. Increased levels of the 
complex IV activity were found (Fig. 4), while interestingly, when considering complex $\mathrm{V}$, its activity increased in MTX22 group and decreased in the MTX48 (Fig. 4). At MTX22, increases in the activity of the complexes IV and $\mathrm{V}$ may be the result of a mitochondrial adaptation to produce more ATP in response to MTX toxic effects. Indeed, in the MTX22 group, no significant differences in the ATP cardiac content compared to control rats were observed (Fig. 5), showing at this time point that the increased activity of the mitochondrial complexes is sufficient to maintain ATP overall levels. However, about one month after the last MTX administration, despite the increase in the complex IV activity, a decrease in complex V activity was observed. In fact, MTX48 rats failed to support the cardiac ATP demand, as observed by decreased cardiac overall ATP levels (Fig. 5). To the best of our knowledge, these results were evidenced for the first time in vivo and were corroborated in vitro (using H9c2 cells) by our research group on ATP-synthase activity using low MTX levels [8]. Conversely, a reduction in the electron transfer activity of state III and a concomitant uncoupling of oxidative phosphorylation were already observed in cardiac mitochondria isolated from MTX-treated female rats ( $2 \mathrm{mg} / \mathrm{kg}$, twice a week, for 4 weeks) [11]. ATP decreased levels can be associated with an inhibited ATP synthesis inhibition (as suggested in this work by the late decreases in the complex V activity), but changes in the ATP levels in the failing myocardium can also be related to the loss of total tissue purines [25], whose levels were not evaluated in the present work.

The mitochondrial effects herein demonstrated in vivo were not observed by the direct incubation of MTX with cardiac mitochondria isolated from untreated rats. The cardiac mitochondria incubated with MTX did not show any change in the mitochondrial functionality evaluated by the distribution of $\mathrm{TPP}^{+}$assay. As already stated, MTX metabolism exerts a pivotal role to the MTX-induced toxicity [7]. Thus, the direct toxic effect mediated by MTX may be residual and insufficient to elicit significant alterations in this in vitro model without previous biotransformation. Moreover, when cardiac mitochondria were isolated from MTX-treated rats and subjected to the $\mathrm{TPP}^{+}$ assay, remarkable heterogeneous results were seen: some animals had fully functional mitochondria, and others presented mitochondria with prolonged lag phase (data not shown). Hence, these data are suggestive that the MTX metabolism seems to be decisive to the toxicity induced by MTX, and differences in the metabolic rate might be behind the intersubject variability observed in the clinical evaluations presented in this work and in human MTX therapy.

In summary, it was demonstrated that MTX induces a relevant cumulative mitochondriopathy evidenced by several indicators of mitochondrial damage: MTX causes aberrant mitochondria and interferes in vivo with the mitochondrial functionality causing an imbalance in the mitochondrial function that results in late ATP depletion and, consequently, a dysfunctional heart. After depletion of its energetic content, the cardiac performance is impaired. Hence, this study highlights the relevance of the MTXinduced mitochondrial toxicity to the MTX-related cardiomyopathy.

Acknowledgments This work was supported by the Fundação para a Ciência e Tecnologia (FCT) - project (EXPL/DTP-FTO/0290/ 2012)-QREN initiative with EU/FEDER financing through COMPETE-Operational Programme for Competitiveness Factors. LGR, VMC, and RJD-O thank FCT for their PhD Grant (SFRH/BD/63473/ 2009) and Post-doc Grants (SFRH/BPD/63746/2009) and (SFRH/ BPD/36865/2007), respectively. The authors are grateful to Fundação para a Ciência e Tecnologia for grant no. Pest C/EQB/LA0006/2011.

\section{References}

1. Seiter, K. (2005). Toxicity of the topoisomerase II inhibitors. Expert Opinion on Drug Safety, 4, 219-234.

2. Kingwell, E., Koch, M., Leung, B., Isserow, S., Geddes, J., Rieckmann, P., et al. (2010). Cardiotoxicity and other adverse events associated with mitoxantrone treatment for MS. Neurology, 74, 1822-1826.

3. Avasarala, J. R., Cross, A. H., Clifford, D. B., Singer, B. A., Siegel, B. A., \& Abbey, E. E. (2003). Rapid onset mitoxantroneinduced cardiotoxicity in secondary progressive multiple sclerosis. Multiple Sclerosis, 9, 59-62.

4. Canal, P., Attal, M., Chatelut, E., Guichard, S., Huguet, F., Muller, C., et al. (1993). Plasma and cellular pharmacokinetics of mitoxantrone in high-dose chemotherapeutic regimen for refractory lymphomas. Cancer Research, 53, 4850-4854.

5. Ehninger, G., Schuler, U., Proksch, B., Zeller, K. P., \& Blanz, J. (1990). Pharmacokinetics and metabolism of mitoxantrone. A review. Clinical Pharmacokinetics, 18, 365-380.

6. Batra, V. K., Morrison, J. A., Woodward, D. L., Siverd, N. S., \& Yacobi, A. (1986). Pharmacokinetics of mitoxantrone in man and laboratory animals. Drug Metabolism Reviews, 17, 311-329.

7. Rossato, L., Costa, V. M., De Pinho, P., Freitas, V., Viloune, L., Bastos, M., et al. (2013). The metabolic profile of mitoxantrone and its relation with mitoxantrone-induced cardiotoxicity. Archives of Toxicology, 10, 1809-1820. doi:10.1007/s00204-013-1040-6.

8. Rossato, L. G., Costa, V. M., Villas-Boas, V., de Lourdes Bastos, M., Rolo, A., Palmeira, C., et al. (2013). Therapeutic concentrations of mitoxantrone elicit energetic imbalance in $\mathrm{H} 9 \mathrm{c} 2$ cells as an earlier effect. Cardiovascular Toxicology. doi:10.1007/ s12012-013-9224-0.

9. Shipp, N. G., Dorr, R. T., Alberts, D. S., Dawson, B. V., \& Hendrix, M. (1993). Characterization of experimental mitoxantrone cardiotoxicity and its partial inhibition by ICRF-187 in cultured neonatal rat heart cells. Cancer Research, 53, 550-556.

10. Alderton, P. M., Gross, J., \& Green, M. D. (1992). Comparative study of doxorubicin, mitoxantrone, and epirubicin in combination with ICRF-187 (ADR-529) in a chronic cardiotoxicity animal model. Cancer Research, 52, 194-201.

11. Bachmann, E., Weber, E., \& Zbinden, G. (1987). Effect of mitoxantrone and doxorubicin on energy metabolism of the rat heart. Cancer Treatment Reports, 71, 361-366. 
12. Padrão, A. I., Ferreira, R. M. P., Vitorino, R., Alves, R. M. P., Neuparth, M. J., Duarte, J. A., et al. (2011). OXPHOS susceptibility to oxidative modifications: The role of heart mitochondrial subcellular location. Biochimica et Biophysica Acta, 1807, $1106-1113$.

13. Pontes, H., Duarte, J. A., De Pinho, P. G., Soares, M. E., Fernandes, E., Dinis-Oliveira, R. J., et al. (2008). Chronic exposure to ethanol exacerbates MDMA-induced hyperthermia and exposes liver to severe MDMA-induced toxicity in CD1 mice. Toxicology, 252, 64-71.

14. Rossato, L. G., Costa, V. M., De Pinho, P. G., Carvalho, F., Bastos, M. L., \& Remião, F. (2011). Structural isomerization of synephrine influences its uptake and ensuing glutathione depletion in rat-isolated cardiomyocytes. Archives of Toxicology, 85, 929-939.

15. Barbosa, D. J., Capela, J. P., Oliveira, J. M., Silva, R., Ferreira, L. M., Siopa, F., et al. (2012). Pro-oxidant effects of ecstasy and its metabolites in mouse brain synaptosomes. British Journal of Pharmacology, 165, 1017-1033.

16. Lowry, O., \& Rosebrough, N. (1951). Protein measurement with the Folin phenol reagent. Journal of Biological Chemistry, 193, 265-272.

17. Rolo, A. P., Oliveira, P. J., Moreno, A. J., \& Palmeira, C. M. (2000). Bile acids affect liver mitochondrial bioenergetics: Possible relevance for cholestasis therapy. Toxicological Sciences, 57, 177-185.

18. Duarte, F. V., Simões, A. M., Teodoro, J. S., Rolo, A. P., \& Palmeira, C. M. (2011). Exposure to dibenzofuran affects lung mitochondrial function in vitro. Toxicology Mechanisms and Methods, 21, 571-576.
19. Patten, R. D., \& Hall-Porter, M. R. (2009). Small animal models of heart failure: Development of novel therapies, past and present. Circulation, 2, 138-144.

20. Novak, R. F., \& Kharasch, E. D. (1985). Mitoxantrone: Propensity for free radical formation and lipid peroxidation-implications for cardiotoxicity. Investigational New Drugs, 3, 95-99.

21. Kharasch, E. D., \& Novak, R. F. (1983). Inhibitory effects of anthracenedione antineoplastic agents on hepatic and cardiac lipid peroxidation. Journal of Pharmacology and Experimental Therapeutics, 226, 500-506.

22. Ingwall, J. S., \& Weiss, R. G. (2004). Is the failing heart energy starved? On using chemical energy to support cardiac function. Circulation Research, 95, 135-145.

23. Ventura-Clapier, R., Garnier, A., \& Veksler, V. (2004). Energy metabolism in heart failure. Journal of Physiology, 555, 1-13.

24. Luft, F. C. (2001). Lactic acidosis update for critical care clinicians. Journal of the American Society of Nephrology, 12, S15-S19.

25. Dzeja, P. P., Redfield, M. M., Burnett, J. C., \& Terzic, A. (2000). Failing energetics in failing hearts. Current Cardiology Reports, 2, 212-217.

26. Kluza, J., Marchetti, P., Gallego, M.-A., Lancel, S., Fournier, C., Loyens, A., et al. (2004). Mitochondrial proliferation during apoptosis induced by anticancer agents: Effects of doxorubicin and mitoxantrone on cancer and cardiac cells. Oncogene, 23, 7018-7030.

27. Khan, S. N., Lai, S. K., Kumar, P., \& Khan, A. U. (2010). Effect of mitoxantrone on proliferation dynamics and cell cycle progression. Bioscience Reports, 30, 375-381. 